\title{
Longer survival in patients with metastatic uterine leiomyosarcoma treated with trabectedin: A case report
}

\author{
THÉOPHRASTE HENRY ${ }^{1}$, ELIZABETH FABRE ${ }^{2}$, LAURENT S. BACCAR ${ }^{3}$ and MICHELE LAMURAGLIA ${ }^{4,5}$ \\ ${ }^{1}$ Assistance Publique-Hôpitaux de Paris, Louis Mourier Hospital, Medical Oncology Unit, \\ Department of Medicine, 92700 Colombes; ${ }^{2}$ Assistance Publique-Hôpitaux de Paris, Hôpital Européen George Pompidou, \\ Department of Medical Oncology, 75908 Paris; ${ }^{3}$ Assistance Publique-Hôpitaux de Paris, Louis Mourier Hospital, \\ Department of Radiology, 92700 Colombes; ${ }^{4}$ Sorbonne Université, CNRS, INSERM, Laboratoire d'Imagerie \\ Biomédicale (LIB), Assistance Publique-Hôpitaux de Paris, Hôpital Beaujon, 75006 Paris; \\ ${ }^{5}$ Assistance Publique-Hôpitaux de Paris, Medical Oncology Unit, Hôpital Beaujon, 92110 Clichy, France
}

Received March 19, 2018; Accepted December 17, 2018

DOI: $10.3892 /$ mco.2019.1797

\begin{abstract}
Trabectedin (ET-743) is a marine alkaloid isolated from the Caribbean tunicate Ecteinascidia turbinata, with a chemical structure characterized by three fused tetrahydroisoquinoline rings. In the present case report, two patients with advanced and metastatic uterine leiomyosarcomas (ULMS) with significant progression-free survival (PFS) and overall survival (OS) administered Trabectedin as second and third line treatment are reported. The first case received third line Trabectedin with a PFS of 24 months and an OS of 35 months. The second case received second line Trabectedin with a PFS of 24 months and an OS of 30 months. In addition, a good safety record was obtained in the long-term administration of Trabectedin (more so in case 1 than case 2), with a good quality of life.
\end{abstract}

\section{Introduction}

Uterine leiomyosarcomas (ULMS) are rare malignant tumors. They represent approximately $1 \%$ of overall uterine tumors, and have a very poor prognosis. The 5-year overall survival (OS) is between 30 and $42 \%$ (1). Less than $20 \%$ of metastatic ULMS showed complete or partial responses with doxorubicin, gemcitabine, or dacarbazine treatment. The median progression-free survival (PFS) was 5 months and OS 12 months $(2,3)$. By contrast, a systematic review has confirmed the potential activity of the combination of gemcitabine

Correspondence to: Dr Michele Lamuraglia, Sorbonne Université, CNRS, INSERM, Laboratoire d'Imagerie Biomédicale (LIB), Assistance Publique-Hôpitaux de Paris, Hôpital Beaujon, UMR 7371, UMR S 1146, 15 Rue de l'école de Médecine (escalier A-3º etage), 75006 Paris, France

E-mail: michele.lamuraglia@aphp.fr

Key words: uterine, leiomyosarcoma, Trabectedin, chemotherapy, metastasis with docetaxel in second line chemotherapy. Benefits of the Trabectedin (Yondelis) are yet to be confirmed (4).

Trabectedin inhibits cell proliferation by specifically binding to the guanine residue within the minor groove of DNA causing a bend in the major groove, which interferes with the DNA binding proteins and transcription factors of the cancer cell. This drug has been shown to have minimal toxicity with a promising PFS and OS (4).

We report two cases demonstrating a durable partial response of Trabectedin in advanced line treatment of patients with advanced and metastatic uterine leiomyosarcoma.

\section{Case report}

Case 1. In July 2010, a 62-year-old female presented pelvic pain. Pelvic echography showed a right tissular latero-uterine mass with cystic component. Pelvic MRI showed a $16 \mathrm{~cm}$ mass, compatible with a necrobiotic myoma. In August 2010, a PET CT-scan performed showed no hypermetabolism and thoracic-abdominal-pelvic CT-Scan (TAP CT-Scan) did not show sign of metastasis. A total hysterectomy associated with omentectomy and appendicectomy was performed on August 2010. Histological analysis revealed the tumor to be a grade 3 uterine leiomyosarcoma. The tumor was $12 \mathrm{~cm}$ long, with high mitotic index (>20 mitotic figures/10HPF). The tumor infiltrated $2 / 3$ of the posterior part of the uterus, and $1 / 3$ of the anterior part. Tumoral invasion was found in the uterine serous, on the surface of the left ovary and in the omentectomy. Tumor necrosis was below $50 \%$. No other local or regional invasion was found. In October 2010, adjuvant radiotherapy was performed. In November 2010, another TAP CT-Scan showed 10 pulmonary micronodules, 2 pulmonary nodules and a right pulmonary embolism. A first line chemotherapy with gemcitabine/docetaxel was initiated in December 2010. Six cycles were performed; however, the dose of docetaxel had to be reduced by $20 \%$ at the second cycle consequence of skin toxicity (skin rash), and then interrupted for the 3 last cycles. TAP CT-Scan performed in June 2011 showed increase in size of the pulmonary nodules with no other progression elsewhere. A second line of chemotherapy with adriamycin/ifosfamide was 
introduced. Six cycles between July 2011 and February 2012 were administered. TAP CT-Scan was performed after 3 cycles and at the end of the six cycles morphological partial response (RECIST criteria) was evident. A therapeutic break was done between February and May 2012. In May 2012, TAP CT-Scan showed progression: increase in size of pulmonary lesions and detection of peritoneal lesions. Therefore, in May 2012, a third line chemotherapy with Trabectedin $\left(1,3 \mathrm{mg} / \mathrm{m}^{2}\right)$ was introduced. After 3 cycles stability in size of the pulmonary and peritoneal nodules was evident. After the sixth cycle, in October 2012, TAP CT-Scan showed partial regression of the peritoneal nodules with stability of the pulmonary lesions. A therapeutic break after the six cycles was performed. Multiple morphological evaluations between October 2012 and June 2013 were performed with persistence of stabile disease RECIST criteria. In June 2013, the TAP CT-Scan showed morphological progression of the pulmonary and peritoneal lesions, resulting in reintroduction of Trabectedin. Nine cycles were performed between June 2013 and May 2014 with morphological stability. Despite the stability in size of the peritoneal lesions the increase in the size of the pulmonary nodules forced us to stop Trabectedin and started a fourth line with weekly Paclitaxel. In November 2014, after progression with Paclitaxel, Pazopanib (Votrien) was started as sixth line. After 3 months of pazopanib, progression led to the introduction of Trabectedin based on the previous relatively good response. In accordance with this long survival of the patient, a new biopsy of pulmonary lesion was made before reintroduction of Trabectedin, which confirmed it was ULMS. Ki-67 that was carried out on this new biopsy was strongly high (70\%), whereas the Ki-67 of the initial biopsy was less important (30\%). Lesions were stable for 6 months with Trabectedin, before presenting another progression and the introduction of best palliative support care. The patient succumbed, in June 2015 (Fig. 1).

Case 2. In September 2012, a 55-year-old female was hospitalized due to asthenia. An abdominal CT-scan was performed and showed multiple suspected malignant hepatic lesions. An already known myomatous uterus with necrobiosis was also described. A biopsy of one of the hepatic lesions allowed us to diagnose a well-differentiated uterine leiomyosarcoma. A PET CT-scan showed multiple hypermetabolic hepatic lesions on the right and left liver, and massive hypermetabolism on the uterine $\left(\mathrm{SUV}_{\max } 12\right)$. Therefore, a hysterectomy with bilateral annexectomy was performed. Histological analysis revealed a ULMS with Ki-67 30-40\%. Annexes were free from tumor, as was the peritoneal cytology. From October to December 2012, 3 cycles of Gemcitabine/Docetaxel were performed, followed by tumoral progression according to RECIST criteria. In fact, TAP CT-Scan showed respectively a 20 and $15 \mathrm{~mm}$ increase of two of the hepatic lesions, and an increase of a peritoneal nodule. From December 2012 to October 2013, 11 cycles of Trabectedin $\left(1.3 \mathrm{mg} / \mathrm{m}^{2}\right)$ were performed. The treatment was not well tolerated, so the patient underwent two major side effects: i) After the first cycle, grade 2 neutropenia was treated by introduction of G-CSF injection; and ii) after the third cycle, an increase of transaminases (grade II), which made us delay the fourth cycle and reduce the chemotherapy dose by $30 \%$. The morphological analyses, performed with TAP
CT-Scan, showed partial response. In February 2013, TAP CT-Scan concluded to a $37 \%$ decrease according to RECIST criteria. Even better, in May 2013, TAP CT-Scan concluded to a subtotal response, where the hepatic lesions were too small to be described, and pulmonary and peritoneal lesions had disappeared (Fig. 2). In October 2013, TAP CT-Scan get a stabilization morphological results obtained to May 2013. A PET-CT scan showed only the hypermetabolic of hepatic lesion in segment VII-VIII $\left(\mathrm{SUV}_{\max } 6.7\right)$ with no other hypermetabolism.

In October 2013, after the thirteenth cycle of Trabectedin, a therapeutic break was started in consequence of very good partial response according to RECIST criteria. In June 2014, after 9 months of therapeutic break morphological progression led to reintroduction of Trabectedin. Partial response was obtained after 3 cycles of treatment, followed by relapse after the sixth cycle. Monotherapy of Ifosfamide was introduced for 3 months without benefit, following 3 months of Pazopanib (Votrien). The continuous progression decreases associated with an alteration of the general state led to introduction of best palliative support care. The patient succumbed, at the end of July 2015 (Fig. 1).

\section{Discussion}

The diagnostic mode employed in the two cases included in this study is noteworthy. It is interesting to see that the discovery of the illness was similar for both of these patients. The findings seemed to be necrotic myoma, whereas they were malignant tumors. New MRI techniques such as diffusion-weighted imaging and apparent diffusion coefficient seem promising to discriminate malignant and non-malignant tumors before surgery (4).

These two cases also show the difficulty involved in the evaluation of the prognosis of uterine leiomyosarcoma. Indeed, the first patient exhibited two major negative prognostic criteria, which is size over $10 \mathrm{~cm}$ and mitotic figures above 20 per HPF (2). According to a previous study, the median survival for grade 3 tumor is 3.1 years (3-5). Nevertheless, the patient of case report 1 is now alive 6 years after her diagnosis.

Adjuvant radiotherapy was performed on the first patient (case 1), who presented a local advanced disease. Nevertheless, studies have failed to confirm the benefit of such a treatment. Its benefit could be increased with subseries of patients with locally advanced disease, and through its combination with chemotherapy to treat metastatic lesions $(6,7)$. Additionally, adjuvant chemotherapy has yet to be proven as beneficial for operating on leiomyosarcoma (5).

It is difficult, especially with only two cases, to determine whether PFS of those patients is linked to natural progrssion of the disease or effect of the chemotherapy. However, Trabectedin was the most well-tolerated drug over a long period of administration for patient case report 1. After 9 months of therapeutic break, disease progression was reduced and the reintroduction of Trabectedin led to approximately 2 years of non-progression. Despite the relatively fast control of the disease obtained with Trabectedin, it has been suggested that it should be administered at least during the first year of therapy, since retarded positive effect has been shown for some patients. The two cases of the current study clearly indicate the 
Overall Survival (OS)

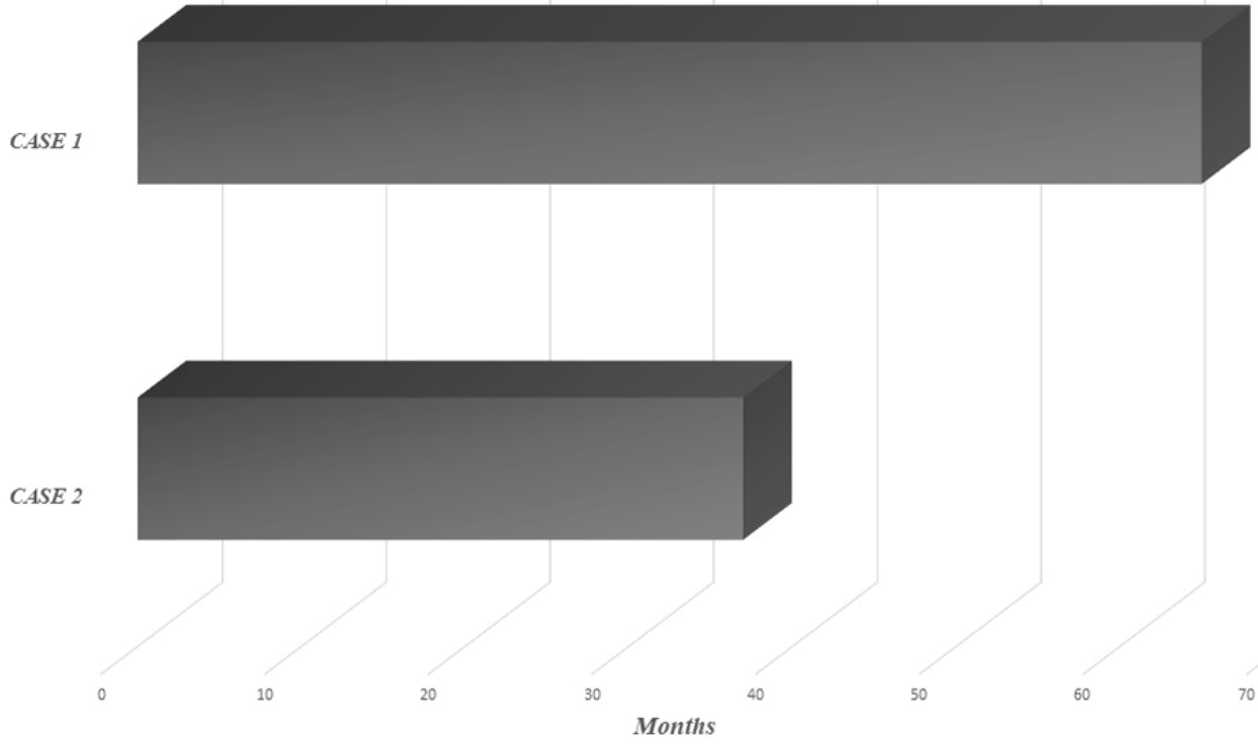

Figure 1. Overall survival of the two patients included in the present case report.
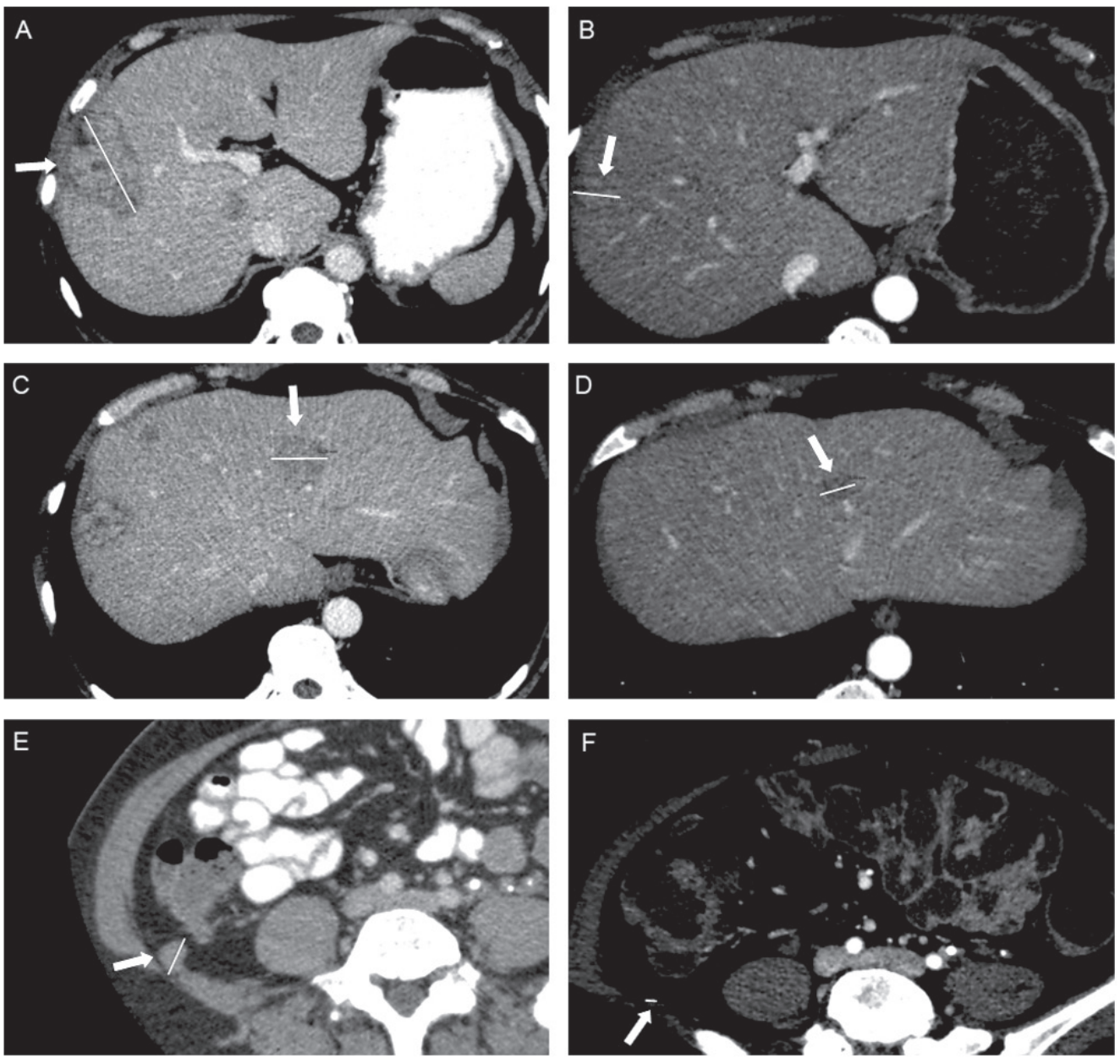

Figure 2. Computed tomography scans of patient 2 exhibiting a partial response following 11 cycles of Trabectedin: Liver lesion of segment VIII-V at (A) $57 \mathrm{~mm}$ vs. (B) $20 \mathrm{~mm}$; liver lesion of segment IVA at (C) $30 \mathrm{~mm}$ vs. (D) $16.6 \mathrm{~mm}$; and peritoneal lesion droit (E) $19 \mathrm{~mm}$ vs. (F) $5 \mathrm{~mm}$ (measurements are indicated by white lines). 
potential of this drug for progressing tumor under first line of chemotherapy. Indeed, findings have shown that despite the fact that Trabectedin has failed to prove its effectiveness in tumor reduction, it seems to have a great stabilization effect, with PFS well above all other types of chemotherapy used for ULMS (8).

In case report 2 , even after heavy dose reduction of Trabectedin, this drug was positively maintained for almost one year and allowed us to obtain a subtotal response. More studies are needed to confirm this stabilization potential; and given the very good tolerance profile of Trabectedin, it is valid to explore concomitant administration with other types of chemotherapy, such as gemcitabine, docetaxel, or doxorubicin. The LMS-02 study, a phase 2 trial, showed promising results for the doxorubicin-trabectedin combination, suggesting synergic effects of these drugs in first-line treatment of metastatic ULM (9).

\section{Acknowledgements}

Not applicable.

\section{Funding}

No funding was received.

\section{Availability of data and materials}

All data generated or analyzed during this study are included in this published article.

\section{Authors' contributions}

TH, ML and EF conceived and designed the manuscript, and collected, analyzed, interpreted and presented the data. AC collected, analyzed and presented the data. LSB contributed to the analysis of all imaging data. All authors have read and approved the final manuscript.

\section{Ethics approval and consent to participate}

Not applicable.

\section{Patient consent for publication}

The patients and their families provided consent for the publication of the present case report.

\section{Competing interests}

The authors declare that they have no competing interests.

\section{References}

1. D'Angelo E, Espinosa I, Ali R, Gilks CB, Rijn Mv, Lee CH and Prat J: Uterine leiomyosarcomas: Tumor size, mitotic index and biomarkers Ki67 and Bcl-2 identify two groups with different prognosis. Gynecol Oncol 121: 328-333, 2011.

2. Italiano A, Mathoulin-Pelissier S, Cesne AL, Terrier P, Bonvalot S, Collin F, Michels JJ, Blay JY, Coindre JM and Bui B: Trends in survival for patients with metastatic soft-tissue sarcoma. Cancer 117: 1049-1054, 2011.

3. Penel N, Van Glabbeke M, Marreaud S, Ouali M, Blay JY and Hohenberger P: Testing new regimens in patients with advanced soft tissue sarcoma: Analysis of publications from the last 10 years. Ann Oncol 22: 1266-1272, 2011.

4. Lin G, Yang LY, Huang YT, Ng KK, Ng SH, Ueng SH, Chao A, Yen TC, Chang TC and Lai CH: Comparison of the diagnostic accuracy of contrast-enhanced MRI and diffusion-weighted MRI in the differentiation between uterine leiomyosarcoma/smooth muscle tumor with uncertain malignant potential and benign leiomyoma. J Magn Reson Imaging 43: 333-342, 2016.

5. Giuntoli RL II, Metzinger DS, DiMarco CS, Cha SS, Sloan JA, Keeney G and Gostout BS: Retrospective review of 208 patients with leiomyosarcoma of the uterus: Prognostic indicators, surgical management and adjuvant therapy. Gynecol Oncol 89: 460-469, 2003.

6. Gupta AA, Yao X, Verma S, Mackay H and Hopkins L; Sarcoma Disease Site Group and the Gynecology Cancer Disease Site Group: Systematic chemotherapy for inoperable, locally advanced, recurrent, or metastatic uterine leiomyosarcoma: A systematic review. Clin Oncol (R Coll Radiol) 25: 346-355, 2013.

7. Gupta AA, Yao X, Verma S, Mackay H and Hopkins L: Chemotherapy (gemcitabine, docetaxel plus gemcitabine, doxorubicin, or trabectedin) in inoperable, locally advanced, recurrent, or metastatic uterine leiomyosarcoma: A clinical practice guideline. Curr Oncol 20: e448-e454, 2013.

8. Monk BJ, Blessing JA, Street DG, Muller CY, Burke JJ and Hensley ML: A phase II evaluation of trabectedin in the treatment of advanced, persistent, or recurrent uterine leiomyosarcoma: A gynecologic oncology group study. Gynecol Oncol 124: 48-52, 2012.

9. Pautier P, Floquet A, Chevreau C, Penel N, Guillemet C, Delcambre C, Cupissol D, Selle F, Isambert N, Piperno-Neumann $\mathrm{S}$, et al: Trabectedin in combination with doxorubicin for first-line treatment of advanced uterine or soft-tissue leiomyosarcoma (LMS-02): A non-randomised, multicentre, phase 2 trial. Lancet Oncol 16: 457-464, 2015. 